\title{
Five-year results of a randomised controlled multi-centre study comparing heavy-weight knitted versus low-weight, non-woven polypropylene implants in Lichtenstein hernioplasty
}

\author{
M. Śmietański • K. Bury • I. A. Śmietańska • R. Owczuk • \\ T. Paradowski · The Polish Hernia Study Group
}

Received: 8 June 2010 / Accepted: 4 March 2011 / Published online: 25 March 2011

(C) The Author(s) 2011. This article is published with open access at Springerlink.com

\begin{abstract}
Background This prospective randomised study evaluated whether non-knitted and non-woven lightweight implants can influence postoperative pain and time of return to normal activity, without increasing the recurrence rate in Lichtenstein inguinal hernioplasty.

Methods Patients were operated on in four centres, randomised blindly into two groups with respect to the mesh used: Surgimesh WN (non-woven polypropylene microfibre sheet) and heavy weight polypropylene mesh. The operation was performed according to the Lichtenstein technique. Follow-up was designed for 7 days, and 3, 6, 12
\end{abstract}

Previous communications: (1) 12 month results of a randomised controlled multi-centre study comparing Prolene and WN mesh in Lichtenstein hernioplasty. European Hernia Society Congress Athens, Greece 2007.

(2) 12 month results of a randomised controlled multi-centre study comparing Prolene and WN mesh in Lichtenstein hernioplasty. Polish Hernia Club Meeting Krakow, Poland 2007.

M. Śmietański $(\bowtie) \cdot$ K. Bury · The Polish Hernia Study Group Department of General and Endocrine Surgery and Transplantation, Medical University of Gdańsk, ul. Dębinki 7, 80-211 Gdańsk, Poland e-mail: smietanski@herniaweb.org

\section{M. Śmietański}

Department of Surgery, District Hospital in Puck, ul. 1-go Maja 13, 84-100 Puck, Poland

I. A. Śmietańska $\cdot$ R. Owczuk

Department of Anesthesiology and Intensive Care,

Medical University of Gdańsk, ul. Dębinki 7,

80-211 Gdańsk, Poland

T. Paradowski

Department of Surgery, City Hospital in Bydgoszcz,

ul. Szpitalna 19, 85-826 Bydgoszcz, Poland and 60 months. The objectives were to assess the incidence of early and late complications, recurrence rate, and postoperative pain.

Results A total of 220 patients were randomised; after monitoring visits (exclusion of one hospital), 202 qualified for the assessment. At 60 months, the recurrence rate did not differ statistically. Less pain on the 7th postoperative day, and at 3 months, was observed in the WN group. No other differences were observed.

Conclusions Use of a lightweight non-woven polypropylene implant is a valuable alternative to the use of knitted or woven meshes in the Lichtenstein method. Postoperative pain and recurrence were reduced at short term follow-up, but no statistical difference in recurrence rate was observed at 12 and 60 month follow-up in the patient population tested.

Keywords Inguinal hernia $\cdot$ Lichtenstein .

Surgimesh WN · Lightweight mesh

\section{Introduction}

In the twenty-first century, mesh implantation has become standard in inguinal hernioplasty. Although many different procedures and mesh types and shapes have been created, the Lichtenstein technique is still the most popular, producing identical results in many surgical centres [1]. The recurrence rate after repair using this technique varies from $<1 \%$ in specialists' hands to $3 \%$ in regional hospitals; however, chronic pain, which has been reported by $>20 \%$ of patients, has become the main postoperative problem associated with this surgery [2,3]. Experimental studies have hypothesised that the inflammatory reaction and scar tissue formation caused by the mesh is responsible for the high incidence of postoperative pain [4]. Material-reduced 
meshes can decrease this inflammation and influence the intensity and rate of pain. Clinical studies on composite (partially absorbable) meshes published in recent years have confirmed this hypothesis [5-9]. All of the materials examined (Vypro, Vypro II, Ultrapro) were used to make macroporous, knitted, partially absorbable meshes of high elasticity. Because of these properties, increased recurrence was noted in one study, but all of the authors were convinced that a dedicated technique for implantation could be designed that would avoid this complication [7]. Furthermore, the role of weight reduction and/or the mesh's macroporous property was not documented as a reason for the decreased rate of pain.

Recently, other materials of reduced weight have been created. The WN mesh that we describe is a low-weight mesh, not knitted or woven, that was designed as a highly porous polypropylene sheet. Until now, this material has not been assessed in randomised trials. Because of its unique design, it allows us to characterise the influence of polypropylene weight reduction on recurrence and pain in the postoperative period.

\section{Patients and methods}

Patients aged between 20 and 75 years who were diagnosed with primary inguinal hernia were eligible to participate in the study. Patients with a history of inguinal hernia repaired with a synthetic mesh, or those on chronic immunosuppressive or corticosteroid therapy, radio- or chemotherapy currently or in the past 3 months, as well as those with chronic renal failure (on dialysis), clinically diagnosed hepatic failure or active bacterial endocarditis, proven mental illness, thrombocytopenia (platelets $<100 \times 10^{9} /$ l) or who were pregnant were excluded from the study. The patients were recruited and operated on in four hospitals in Poland. All patients gave informed consent. The study was approved by the Ethical Committee of the Medical University of Gdansk for all participating centres, and registered in the Clinical Trials Database of Medical University of Gdansk in accordance with Polish law (approval and registration number: NKEBN/872/3003).

Operative technique and anaesthesia

The standard operative technique by Lichtenstein and Amid was used in both groups [2]. In the control group (called the HW PP group), heavyweight polypropylene mesh was implanted (PROLENE, Ethicon GMbH, Hamburg, Germany). In the treated group (the WN group), a lowweight (approximately $38 \mathrm{~g} / \mathrm{m}^{2}$ ), non-woven, non-knitted implant made from polypropylene microfibres was used (Surgimesh $\mathrm{WN}^{\circledR}$, Aspide Medical, France) (Fig. 1).

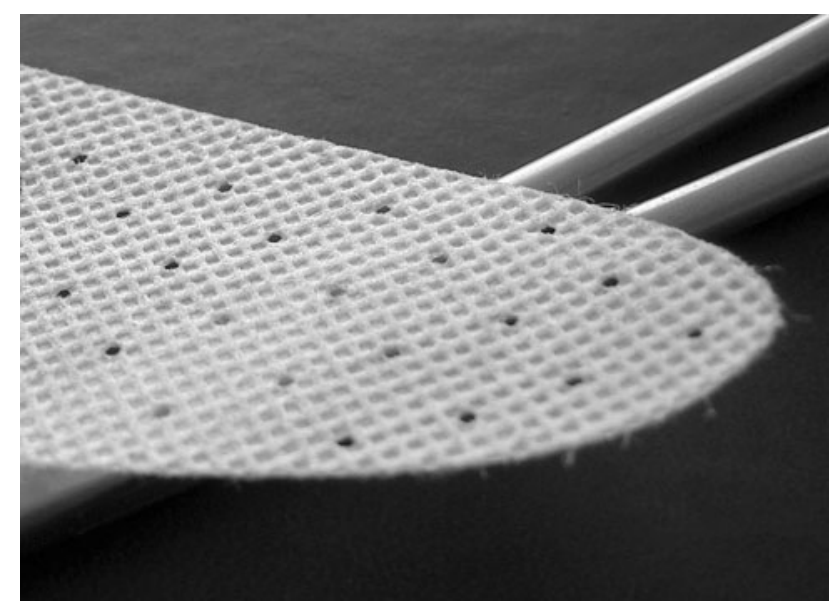

Fig. 1 Surgimesh: a low-weight, non-woven, non-knitted implant made from polypropylene microfibre

In both the WN and HW PP groups, Prolene 2-0 was used for mesh implantation. Cefazolin $(1 \mathrm{~g})$ was administered intravenously (IV) $0.5 \mathrm{~h}$ before the operation as antibiotic prophylaxis. Standardised anaesthesia was applied for all patients. A subdural block was effected with $4 \mathrm{ml}$ bupivacaine hydrochloride (Marcaine Spinal 0.5\% Heavy, Astra Zeneca Pharma) using a pencil point needle (27 G). In addition, the amount of fluid given in the postoperative period was standardised (IV crystalloid: $2 \mathrm{ml} \mathrm{kg}^{-1} \mathrm{~h}^{-1}$ from the last drink taken until the end of the operation; after the operation, a minimum of 1.51 fluid by mouth). Four hours after the operation, patients were allowed to stand up; after $6 \mathrm{~h}$, they could be discharged.

\section{Objectives and outcomes}

We hypothesised that the WN mesh would cause less pain at short- and long-term observations. In this trial, we studied patients at six controlled visits over 1 year (at hospital stay and postoperatively at 7 days, 3 months, 6 months, and 1 and 5 years). Additionally, hernia characteristics, time of occurrence, and preoperative pain were assessed. The 5-year observation was designed to assess long-term results (recurrence rate and chronic pain). All visits were conducted at each respective hospital. Physical examinations in the postoperative period were performed by a surgeon who had not been involved in the operation and who was blinded to the type of mesh used. Postoperative pain and quality of life questionnaires were administered by a nurse or student who was also blinded to the type of mesh. The primary endpoint of the study was presence of pain or hernia recurrence. Acute pain was defined as pain reported by a patient within the first 6 months after the operation. Pain after 12 months was defined as "chronic", following the definition given by Kehlet et al. [9]. Recurrence was noted based on a surgeon's examination and diagnosis. Presence 
of pain and recurrence were assessed on every visit scheduled in the study flow chart. Additionally, the following data were collected during the hospital stay and until 3 months later: perioperative complications (haematoma, seroma, nerve damage), urinary retention, need for urinary catheter placement and wound infection rate.

\section{Study flow chart}

Four hospitals were included in the trial according to their capacity for patients and experience with the Lichtenstein technique (a minimum of 150 inguinal hernias/year, and a minimum of 200 Lichtenstein procedures done by the investigator). Before patients were recruited, a workshop on the operative technique for the investigational mesh was conducted for all trial participants. A total of 220 patients were randomised in the trial. The randomisation scheme was generated using the website randomisation.com (http:// www.randomization.com), which utilises the pseudo-random number generator of Wichmann and Hill, modified by McLeod. Subjects were divided into groups of equal size, which were distributed to each hospital prior to patient inclusion. Three months after randomisation had ended, monitoring visits in all centres were conducted by members of the steering committee. The data were double-checked by comparing patient study files versus hospital documentation and against random patient personal reports (via visits or telephone examination) (Fig. 2).

\section{Statistical analysis}

The minimum number of patients in each study group was calculated based on the assumption that reduction of pain incidence from $20 \%$ to $30 \%$ (as observed earlier in individuals with polypropylene mesh) to $10 \%$ in the first 3 months of observation would be clinically significant. With this assumption, a test power of $80 \%$, and an alpha level of 0.05 , we calculated that 100 patients were needed in each study group. The final patient number was 110 per group due to anticipation of possible patient "losses" (see "Study flow chart"). This number of patients corresponded with the trials (available at the time of study design) by Post or Kinsnorth that were designed to assess new materials (lightweight polypropylene) or devices (PHS, Mesh-Per-fix-plug) [10]. Statistical calculations were performed using Statistica 7.1 PL (Polish version) software (StatSoft, Tulsa, OK). Descriptive statistics were used to characterise patient groups, and mean (standard deviation) or median values (range of values) were given, depending on the type of data and a normal data distribution in the interval scale. Normal distributions were verified with the W Shapiro-Wilk's test. Data were compared using Student's $t$ test or the Mann-Whitney $U$ test. Repeated measurements were analysed using a two- way analysis of variance (ANOVA) test for repeated measurements, with subsequent analysis of significant differences with the post-hoc method (HSD Tukey's test) when appropriate. Categorical data were presented as percentages and $95 \%$ confidence intervals and compared using a chisquare test, with the Yates correction applied when necessary. A significance level of $P<0.05$ was adopted.

\section{Results}

Of all the patients randomised after monitoring visits, 101 patients in the WN group and 99 in the HW PP group were included in the trial database. Baseline data for both groups are presented in Table 1. After randomisation, statistical differences was noted between the groups according to hernia size (medium indirect hernias were often presented in the WN group, smaller indirect in the HW PP group). From these patients, 12-month follow-up examinations were completed by $98.5 \%$ ( 3 patients were lost to follow-up), respectively, and 5-year follow-up was completed by $90.1 \%$ (an additional 17 patients lost to follow-up).

The median operative time was $40 \mathrm{~min}$ (15-125 $\mathrm{min}$ ) for the WN group and $35 \mathrm{~min}(20-95 \mathrm{~min})$ for the HW PP group $(P=0.43)$. No serious perioperative or postoperative complications were noted in either group, and there were no statistically significant differences between the groups, except that wound redness (1\% vs $8.1 \% ; P=0.013)$ and superficial haematoma ( $1 \%$ vs $7 \% ; P=0.07)$ were often noted in the HW PP group (Table 2). Postoperative measurements revealed a significantly higher frequency of pain in the HW PP group in early visits ( 7 days and 3 months) (Table 3). However, although this difference was also noted after 6 months and 1 year, it was not significant due to the small number of patients suffering from pain in both groups $(<10 \%)$, No difference was noted at the 5-year observation point (Table 3 ). The intensity of pain measured in VAS (data were counted for the whole group, not only for the patients with pain) was also lower in the WN group, although a statistical difference was found only 7 days postoperatively $(P=0.0012)$. It should be mentioned that the intensity of pain was slightly higher in the HWPP group before surgery, but this difference was not statistically significant. In addition, pain after 7 days did not differ from the baseline for the HW PP group, as opposed to the WN group, in which it was statistically lower $(P=0.0006)$. In both groups, the intensity of pain measured for the whole group was significantly lower after 3 months compared to baseline (all $P$ values $<0.05$ ). Statistical analysis of the intensity of pain (VAS) was not possible to calculate for the whole population after 3 months due to the small number of patients suffering from pain. Intensity of pain in the first 3 months is presented in Fig. 3 . 


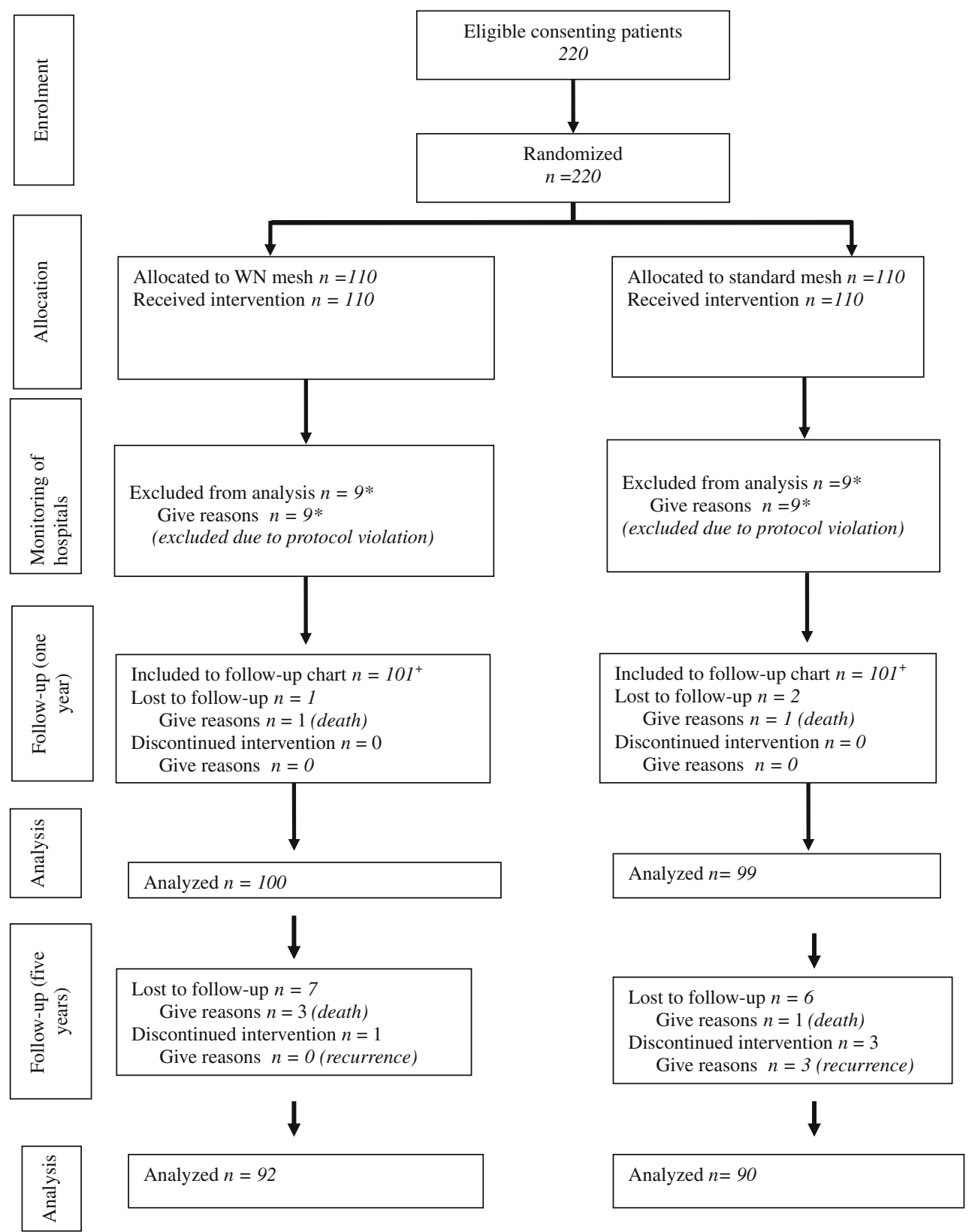

Fig. 2 Randomized trial of WN or polypropylene mesh in Lichtenstein primary inguinal hernia repair. + Included into final analysis after monitoring visits in the hospitals, * excluded after monitoring visits in hospitals—see text

The incidence of recurrence was slightly higher in the HW PP group (3\%) than in the WN group (1\%) at 5 years, but no statistical difference was found $(P=0.61)$. All recurrences were in male patients and occurred within the first 2 years of follow-up. In all cases, the recurrence was found above the pubic bone at the mesh margin.

\section{Discussion}

In this study, a newly introduced implant of a different design resulted in an improved pain profile and lower recurrence rate at 60-month follow-up, compared to rates for the knitted polypropylene traditionally used for Lichtenstein hernioplasty. We have confirmed that this mesh may be used in a routine clinical setting as the first-choice mesh for inguinal hernia repair. This multicentre trial was created based on our previous findings from a small pilot study that indicated the possible advantages of this implant [11]. Previously, we encountered less pain with WN versus heavyweight polypropylene mesh, and no recurrences in the $\mathrm{WN}$ mesh group. The group treated in that study was quite small (25 patients in each cohort), so those differences might have been coincidental. Based on those results, it was mandatory to design and conduct a larger trial. 
Table 1 Patient baseline data

\begin{tabular}{|c|c|c|c|}
\hline & $\begin{array}{l}\text { Non-woven WN, } \\
(n=100)\end{array}$ & $\begin{array}{l}\text { HW PP, } \\
(n=99)\end{array}$ & $P$ value \\
\hline Age (years) & $55.3 \pm 14.9$ & $58.5 \pm 13.7$ & $0.17^{\mathrm{a}}$ \\
\hline Weight $(\mathrm{kg})$ & $76.8 \pm 10.5$ & $75.8 \pm 11.0$ & $0.56^{\mathrm{a}}$ \\
\hline Height $(\mathrm{cm})$ & $174(152-182)$ & $173(158-185)$ & $0.58^{\mathrm{b}}$ \\
\hline \multicolumn{4}{|c|}{ Type of hernia (Rutkow classification)_EHS classification (\% and nr of patients) } \\
\hline 1 (indirect—normal deepening ring)—L1 & $3(3.0 \%)$ & $18(18.2 \%)$ & \multirow[t]{6}{*}{$0.006^{\mathrm{c}}$} \\
\hline 2 (indirect—dilated ring $<4 \mathrm{~cm}$ ) - $\mathrm{L} 2$ & $45(45.0 \%)$ & $35(35.4 \%)$ & \\
\hline 3 (indirect-ring $>4 \mathrm{~cm}$ ) - $\mathrm{L} 3$ & $21(21.0 \%)$ & $25(25.3 \%)$ & \\
\hline 4 (direct-large defect of the canal floor) M2 or 3 & $24(24.0 \%)$ & $12(12.1 \%)$ & \\
\hline 5 (direct—small medial orifice)-M1 & $4(4.0 \%)$ & $5(5.1 \%)$ & \\
\hline 6 (combined direct and indirect) $-\mathrm{L} 1 \mathrm{M}$ & $3(3.0 \%)$ & $4(4.1 \%)$ & \\
\hline Pain before operation (days) & $3(0-240)$ & $5(0-200)$ & $0.013^{\mathrm{b}}$ \\
\hline Duration of operation (min) & $40(15-125)$ & $35(20-95)$ & $0.43^{\mathrm{b}}$ \\
\hline Time from hernia occurrence to operation (days) & $6(0.5-240)$ & $6(0.5-480)$ & $0.52^{\mathrm{b}}$ \\
\hline Pain before operation ( $n, \%$ of population and $95 \% \mathrm{CI}$ ) & $67,67.0 \%(57.3-75.4)$ & $62,62.6 \%(52.8-71.6)$ & $0.52^{\mathrm{d}}$ \\
\hline
\end{tabular}

${ }^{\text {a }}$ Compared using Student's $t$ test

b Compared using $U$ Mann-Whitney's test

c Calculated with chi-square test

d Calculated with chi-square test with Yates correction

Table 2 Peri- and post-operative complications and analgesics intake in both groups. Data are presented as $n$ [\% and (CI)], all comparisons calculated with chi-square test or with chi-square test with Yates correction

\begin{tabular}{|c|c|c|c|}
\hline Assessed value & Non-woven WN, $(n=100)$ & HW PP, $(n=99)$ & $P$ value \\
\hline Perioperative nerve injury & $0[0 \%(0-3.7)]$ & $1[1 \%(0.2-5.45)]$ & 1.0 \\
\hline Analgesics consumption on 1st postoperative day & $90[90 \%(82.6-94.5)]$ & $91[95 \%(84.9-95.9)]$ & 0.64 \\
\hline Superficial haematoma & $1[1 \%(0.2-5.45)]$ & $7[7.1 \%(3.5-13.9)]$ & 0.07 \\
\hline Urine retention & $0[0 \%(0-3.7)]$ & $1[1 \%(0.2-5.45)]$ & 1.0 \\
\hline Need for urinary catheter placement & $1[1 \%(0.2-5.45)]$ & $1[1 \%(0.2-5.45)]$ & 1.0 \\
\hline Redness of wound or wound edema & $1[1 \%(0.2-5.45)]$ & $8[8.1 \%(4.2-15.1)]$ & 0.039 \\
\hline Wound infection & $0[0 \%(0-3.7)]$ & $0[0 \%(0-3.7)]$ & 1.0 \\
\hline Recurrence after 12 months & $0[0 \%(0-3.7)]$ & $0[0 \%(0-3.7)]$ & 1.0 \\
\hline Recurrence after 60 months & $1[1 \%(0.2-5.45)]$ & $3[3 \%(1.0-8.50]$ & 0.61 \\
\hline
\end{tabular}

Table 3 Patients suffering for pain in examined groups. Data are presented as $n[\%$ and $(\mathrm{CI})]$

\begin{tabular}{llll}
\hline Visit & $\begin{array}{l}\text { Non-woven WN, } \\
(n=100)\end{array}$ & HW PP, $(n=99)$ & $P$ value \\
\hline Preoperatively & $67[67 \%(57.3-75.4)]$ & $62[62.6 \%(52.8-71.5)]$ & $0.52^{\mathrm{a}}$ \\
7 days & $42[42 \%(32.8-51.8)]$ & $55[55.6 \%(45.7-64.9)]$ & $0.06^{\mathrm{a}}$ \\
3 months & $16[16 \%(10.1-24.4)]$ & $23[23.2 \%(16.0-32.5)]$ & $0.19^{\mathrm{a}}$ \\
6 months & $5[5 \%(2.2-11.2)]$ & $10[10.1 \%(5.6-17.6)]$ & $0.28^{\mathrm{b}}$ \\
12 months & $6[6 \%(2.8-12.5)]$ & $8[8.1 \%(4.15-15.1)]$ & $0.57^{\mathrm{a}}$ \\
60 months & $2[2 \%(0.6-7.0)]$ & $2[2 \%(0.6-7.1)]$ & 1.0 \\
\hline
\end{tabular}

${ }^{a}$ Calculated with chi-square test

${ }^{\mathrm{b}}$ Calculated with chi-square test with Yates correction
In recent decades, many studies had proven that use of mesh decreases acute pain (up to 6-12 months) compared to tension methods. However, the incidence and intensity of postoperative pain are still high, affecting up to $20 \%$ of patients operated on with mesh $[12,13]$. To reduce this problem, companies are searching for new materials that can potentially reduce the number of patients suffering from pain due to the presence of a foreign body after the procedure. Experimental studies have shown that the extent of reaction to a foreign body, with the scar tissue and inflammatory reaction it provokes, depends on the amount and structure of the material implanted [4]. New meshes have been introduced into the market. An absorbable component 


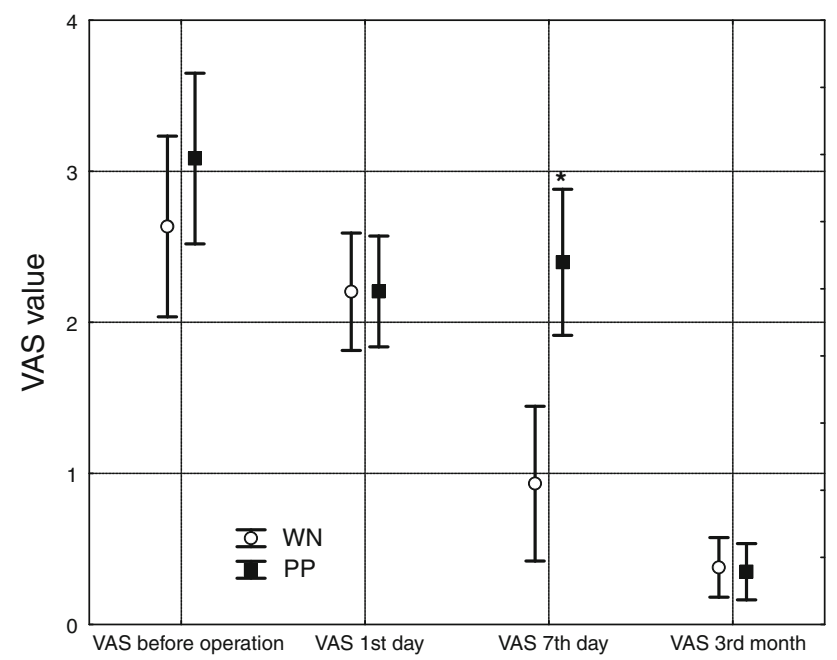

Fig. 3 VAS score in follow-up period (means and 95\% confidence intervals)—compared with ANOVA and post-hoc HSD Tukey's tests. $* P=0.0012$ when compared with VAS value in $\mathrm{WN}$ group at the same time point

has been added to the polypropylene fibres to decrease the weight of mesh during the incorporation process, and the potential advantage conferred by partially absorbable lightweight meshes, such as Vypro, Vypro II and Ultrapro, has been confirmed in clinical studies conducted by Post, O'Dwyer and the Polish Hernia Study Group [5, 6, 8]. The incidence of pain of any sort was generally two times lower in lightweight mesh groups, but the values ranged from $39 \%$ [5] to $3.8 \%$ [8] for the LW meshes, and from $51 \%$ to $6.8 \%$ in the HW groups, respectively. On the other hand, Bringman found no significant difference in pain scores when a Vypro II implant was compared with polypropylene mesh in another large study (only the pain occurring when rising from a lying to a sitting position was lower in the lightweight mesh group) [7]. There is no meta-analysis of the above-mentioned trials, and the values measured seems to differ due to the different protocols used for assessment of pain. In our present study, early postoperative pain depended on the mesh used. These trends were also noted over the first 3 months, but the small number of patients precluded definitive statistical evaluation. However, the incidence of pain observed here was comparable to pain from lightweight meshes, which may also confirm the findings of other trials $[8,14]$. For other meshes (low weight partially absorbable meshes), the tissue reaction and fibroblastic ingrowth creates a scar that is responsible for mesh shrinkage; this has been described in experimental studies [4]. Formation of an elastic scar on megaporous meshes should theoretically prevent pain and/or a feeling of a foreign body in the groin. These differences in pathophysiological processes have not been observed for the WN implant. Experimental studies have shown no statistical differences in visual sign of inflammatory response (electron-microscope measurements), fibrotic reaction and implant integration for non-woven mesh compared to HW knitted polypropylene [15]. Only the total amount of macrophages at the site of the mesh was statistically higher in the non-woven mesh group, which supports the assumption of a more pronounced inflammatory reaction caused by the mesh structure [15].

In the last few decades, recurrence has been shown to be an outcome of less value than pain, due to an incidence below $1-2 \%$ in $3-5$ years of follow-up. Introduction of lightweight meshes has again focussed attention on this complication. In most studies, the recurrence rate was significantly higher in the lightweight mesh groups, being as high as 5\% [6]. The authors of the O'Dwyer's trial suggested that the high rate of recurrence may be a consequence of the different elastic properties of Vypro II mesh, and could require modification of the fixation technique. The modification of the operative technique postulated by O'Dwyer and applied by the Polish Hernia Study Group has reduced the recurrence rate to accepted levels of about $2 \%$ in first year of observation $[8,16]$. Although the rate noted in the present study was less than $2 \%$ after 1 year for the HW implant, it was lower still in the non-woven WN group. Additionally, the WN mesh described here does not require any modification of the classical Lichtenstein technique due to its firmness and shape memory. We did not find any differences when handling and suturing this implant. In this study, as in the pilot study, we did not observe any recurrences in the WN group up to 1 year, and very low recurrence rates after 5 years.

Summarizing the described findings, we conclude that the examined lightweight implant shows good results in preventing postoperative pain, being comparable with other low weight materials and superior to HW mesh. Also, the low recurrence rate at 5-year follow-up confirms the value of this implant, especially considering that long-term results have not been described for other LW materials to date. WN mesh can be safely considered as an appropriate choice of implant for primary inguinal mesh repair.

Acknowledgments The study was supported by a minor grant from Inter Consult Poland to cover the costs of workshops and trialists meetings. Neither physicians fees nor patients reimbursement were provided. None of the authors has any direct or indirect financial interest in the product.

Members of Polish Hernia Study Group involved in the trial:

M. Śmietański (project leader)

Steering committee: I.A. Śmietańska (consultant anesthesiology), K.Bury (data base management, monitoring menager), R.Owczuk (consultant statistics), T.Paradowski (protocol design, operative technique quality control management)

Members (patients enrolment, data collection): T. Kạtny (Bydgoszcz), A.Olejarz (Bydgoszcz), A. Marczynski (Bytom),W. Niewiadomski (Bytom), J. Marczynski (Bytom), A. Spilarewicz (Świdnica), A. Biernacki (Świdnica), M. Wilkos (Świdnica), J.Przydatka (Biała Podlaska), K. Horoszewicz (Biała Podlaska), Z Kamiński (Biała Podlaska). 
Open Access This article is distributed under the terms of the Creative Commons Attribution Noncommercial License which permits any noncommercial use, distribution, and reproduction in any medium, provided the original author(s) and source are credited.

\section{References}

1. EU Hernia Trialists Collaboration (2000) Mesh compared with non-mesh methods of open groin hernia repair: systematic review of randomized controlled trials. Br J Surg 87:845-859

2. Amid P (2003) Lichtenstein repair in 2002: an overview of causes of recurrence after Lichtenstein tension-free hernioplasty. Hernia 7:13-16

3. Callesen T, Bech K, Kehled H (1999) Prospective study of chronic pain after groin hernia repair. Br J Surg 86:1528-1531

4. Klinge U, Klosterhalfen B, Muller M, Schumpelick V (1999) Foreign body reaction to meshes used for the repair of abdominal wall hernias. Eur J Surg 165:665-673

5. Post S, Weiss B, Willer M, Neufang T, Lorenz D (2004) Randomized clinical trial of lightweight composite mesh for Lichtenstein inguinal hernia repair. Br J Surg 91:44-48

6. O'Dwyer PJ, Kingsnorth AN, Molloy RG, Small PK, Lammers B, Horeyseck G (2005) Randomized clinical trial assessing impact of a lightweight or heavyweight mesh on chronic pain after inguinal hernia repair. Br J Surg 92:166-170

7. Bringman S, Wollert S, Osterberg J, Smedberg S, Granlund H, Haikkinen T-J (2006) Three-year results of a randomized clinical trial of lightweight or standard polypropylene mesh in Lichtenstein repair of primary inguinal hernia. Br J Surg 93:1056-1059

8. Polish Hernia Study Group (2008) Randomized controlled multicentre study comparing a polypropylene and poliglecaprone/ polypropylene composite mesh for inguinal hernioplasty. Br J Surg 95:1462-1468

9. Kehlet H, Bay-Nielsen M, Kingsnorth AN (2002) Chronic postherniorrhaphy pain —a call for uniform assessment. Hernia 6:178181

10. Kingsnorth AN, Porter CS, Bennet DH, Waller AL, Hyland ME, Sodergren S (2000) Lichtenstein patch or Perfix plug-and-patch in inguinal hernia: a prospective double-blind randomized controlled trial of short-term outcome. Surgery 127:276-283

11. Paradowski T, Olejarz A, Kontny T, Łukasiewicz J, Śledziński Z, Śmietańska IA, Śmietański M (2009) Polypropylene vs ePTFE vs WN mesh for Lichtenstein inguinal hernia repair-a prospective, randomized, double blind pilot study of one-year follow-up. Articles Videosurg Miniinvasive Tech 4(1):6-9

12. van Veen RN, Wijsmuller AR, Vrijland WW, Hop WC, Lange JF, Jeekel J (2007) Long-term follow-up of a randomized clinical trial of non-mesh versus mesh repair of primary inguinal hernia. $\mathrm{Br} \mathrm{J}$ Surg 94:506-510

13. van Veen RN, Wijsmuller AR, Vrijland WW, Hop WC, Lange JF, Jeekel J (2007) Randomized clinical trial of mesh versus non-mesh primary inguinal hernia repair: long-term chronic pain at 10 years. Surgery 142(5):695-698

14. Paajanen H (2007) A single-surgeon randomized trial comparing three composite meshes on chronic pain after Lichtenstein hernia repair. Hernia 11:335-339

15. Weyhe D, Schmitz I, Belyaev O, Grabs R, Müller KM, Uhl W, Zumtobel V (2006) Experimental comparison of monofile light and heavy polypropylene meshes: less weight does not mean less biological response. World J Surg 30(8):1586-1591

16. Smietański M, Bigda J, Zaborowski K, Worek M, Sledziński Z (2009) Three-year follow-up of modified Lichtenstein inguinal hernioplasty using lightweight poliglecaprone/polypropylene mesh. Articles Videosurg Miniinvasive Tech 13(3):239-242 